\title{
Norovirus epidemiology in South African children $<5$ years hospitalised for diarrhoeal illness between 2009 and 2013
}

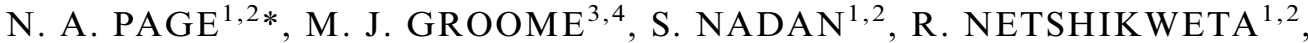 \\ K. H. KEDDY ${ }^{1,5}$, B. POONSAMY ${ }^{1}, \mathrm{~J}_{\text {. MOYES }}{ }^{6}, \mathrm{~S} . \mathrm{WALAZA}^{1}, \mathrm{~K} \cdot \mathrm{KAHN}^{7}$, \\ S. A. MADHI ${ }^{1,3,4}$, M. B. TAYLOR ${ }^{2}$, J. MANS $^{2}$ AND C. COHEN ${ }^{1}$ \\ ${ }^{1}$ National Institute for Communicable Diseases, Private Bag x4, Sandringham, 2131, South Africa \\ ${ }^{2}$ Department of Medical Virology, Faculty of Health Sciences, University of Pretoria, Private Bag X323, \\ Arcadia, Pretoria 0007, South Africa \\ ${ }^{3}$ Medical Research Council: Respiratory and Meningeal Pathogens Research Unit, University of the \\ Witwatersrand, Johannesburg, South Africa \\ ${ }^{4}$ Department of Science and Technology/National Research Foundation: Vaccine Preventable Diseases, \\ University of Witwatersrand, Johannesburg, South Africa \\ ${ }^{5}$ Clinical Microbiology and Infectious Diseases, Faculty of Health Sciences, University of Witwatersrand, \\ Johannesburg, South Africa \\ ${ }^{6}$ School of Public Health, Faculty of Health Sciences, University of Witwatersrand, Johannesburg, South Africa \\ ${ }^{7}$ MRC/Wits Rural Public Health and Health Transitions Research Unit (Agincourt), School of Public Health, \\ Faculty of Health Sciences, University of Witwatersrand, Johannesburg, South Africa
}

Received 9 September 2016; Final revision 3 March 2017; Accepted 14 March 2017; first published online 10 April 2017

\section{SUMMARY}

Public health interest in norovirus (NoV) has increased in recent years following improved diagnostics, global burden estimates and the development of NoV vaccine candidates. This study aimed to describe the detection rate, clinical characteristics and environmental features associated with NoV detection in hospitalized children $<5$ years with diarrhoea in South Africa (SA). Between 2009 and 2013, prospective diarrhoeal surveillance was conducted at four sites in SA. Stool specimens were collected and screened for NoVs and other enteric pathogens using molecular and serological assays. Epidemiological and clinical data were compared in patients with or without detection of NoV. The study detected NoV in $15 \%$ (452/3103) of hospitalized children $<5$ years with diarrhoea with the majority of disease in children $<2$ years $(92 \% ; 417 / 452)$. NoV-positive children were more likely to present with diarrhoea and vomiting (odds ratio (OR) $1 \cdot 3 ; 95 \%$ confidence interval (CI) $1 \cdot 1-1 \cdot 7 ; P=0 \cdot 011$ ) with none-to-mild dehydration (adjusted OR $0 \cdot 5 ; 95 \%$ CI 0-3-0·7) compared with NoV-negative children. Amongst children testing NoV positive, HIV-infected children were more likely to have prolonged hospitalization and increased mortality compared with HIV-uninfected children. Continued surveillance will be important to consider the epidemic trends and estimate the burden and risk of NoV infection in SA.

Key words: Caliciviruses, diarrhoea, Norwalk agent and related viruses, virology (human) and epidemiology.

\footnotetext{
* Author for correspondence: N. Page, National Institute for Communicable Diseases, Private Bag x4, Sandringham, 2131, South Africa and Department of Medical Virology, Faculty of Health Sciences, University of Pretoria, Private Bag X323, Arcadia, Pretoria 0007, South Africa. (Email: nicolap@nicd.ac.za)

Permanent address: Centre for Enteric Diseases, National Institute for Communicable Diseases, Private Bag x4, Sandringham, Johannesburg 2131, South Africa.
} 


\section{INTRODUCTION}

Globally, norovirus (NoV) are a common cause of viral diarrhoea and vomiting in children $<5$ years [1]. Awareness of NoV has heightened with the improvement of diagnostics to detect the virus and the identification of NoV as an important cause of food-borne illness globally [2, 3]. A systematic review of NoV in sporadic gastroenteritis cases conducted in 2014 estimated that NoV was responsible for 18\% (95\% confidence interval (CI) 15-20) of gastroenteritis among children $<5$ years [1]. A previous review in 2008 estimated that NoV was responsible for $12 \%$ (95\% CI 10-15) of severe diarrhoea in children $<5$ years and projected 218000 deaths in children $<5$ years in developing countries annually [4].

While NoV infections are generally self-limiting, outcomes can be severe in individuals with comorbidities, extremes of age ( $<5$ and $>65$ years) or following the emergence of new strains [5, 6]. A study conducted in the USA demonstrated that children $<5$ years have the highest rates of NoV-associated health care visits and adults $\geqslant 65$ years have the greatest risk of death due to NoV [7]. NoV disease in children is generally mild, however, severe outcomes do occur with hospitalization rates between $7 \cdot 2$ and 16 hospitalizations/ 10000 population $<5$ and an estimated $9 \cdot 9-12 \%$ of diarrhoeal deaths annually [7, 8].

Mans et al. recently conducted a review of NoV epidemiology in Africa [9]. While data on NoV prevalence in patients with diarrhoea in sub-Saharan Africa are available, most of these studies have duration of $<2$ years or $<300$ specimens available for evaluation. Despite these limitations, the review revealed overall NoV prevalence in individuals with diarrhoea of $13 \cdot 5 \%(961 / 7141 ; 95 \%$ CI $12 \cdot 7-14 \cdot 3)$, with children $<1$ year mainly affected in African settings [9].

Research on NoV in South Africa (SA) dates back to 1993 [10] and originally focused on seroprevalence rates in all age groups, which ranged from $>50 \%$ to $>90 \%[11,12]$. An early virus detection study applied a combination of electron microscopy and recombinant enzyme immunoassays against Norwalk (NV)-like and Mexico (MX)-like NoVs and RTPCR (reverse transcription-polymerase chain reaction) for the detection of caliciviruses in paediatric stool specimens collected between 1991 and 1995 [13]. NV-like and MX-like NoVs were detected in $0 \cdot 2 \%(3 / 1296)$ and $2 \cdot 2 \%(29 / 1296)$ specimens, respectively [13].
Between November 2009 and February 2012, the multisite birth cohort study investigating pathogenspecific burdens of community diarrhoea (MAL-ED) included a site in Venda, SA [14]. NoV incidence at this site was $9 \cdot 52(95 \%$ CI $7 \cdot 06-12 \cdot 83)$ NoV GII detected per 100 child-months and 5.09 NoV GI detected per 100 child-months [14]. Severity of NoV diarrhoea was comparable with other enteric pathogens detected, except RV, and undernutrition was identified as a risk factor for NoV disease [14]. NoV infections peaked in children 6-11 months of age [14].

In immunocompromised hosts, NoV infections are reported to be more severe with prolonged symptoms and viral shedding [6]. However, the majority of these studies have been conducted in transplant recipients with limited information in HIV-infected individuals [6]. Persistent diarrhoea, lasting 7 months, was described in an HIV-infected adult patient with poor compliance to antiretroviral therapy and a chronic NoV infection [15]. NoVs were detected more often in HIV-infected compared with HIV-uninfected children [16, 17].

In April 2009, a prospective sentinel surveillance system was established to monitor diarrhoeal disease in hospitalized children $<5$ years of age in SA. We aimed to describe the epidemiology of NoV between 2009 and 2013.

\section{METHODS}

\section{Study participants and sites}

Hospitalized children $<5$ years with acute diarrhoea were enrolled in the sentinel diarrhoea surveillance study. Acute diarrhoea was defined according to the World Health Organization (WHO) definition of 'three looser than normal stools within a $24 \mathrm{~h}$ period'. The sentinel sites, located in three provinces, included: Chris Hani Baragwanath Academic Hospital (CHBAH; 2009-2013), Mapulaneng Hospital (MPH; 2009-2013), Matikwane Hospital (MKH; 2009-2013) and Edendale Hospital (EDH; 2010-2013).

\section{Study enrolment and data collection}

Written informed consent was obtained from the parents of patients prior to enrolment. Systematic sampling was used to enrol patients on a daily basis from Monday to Friday between 8 am and $5 \mathrm{pm}$. Patients who refused to participate in the study were noted, but no additional information was collected. Surveillance officers collected demographic, socio-economic and risk factor data from 
the parents by interview and additional clinical data from medical records on standardized questionnaires. Data on HIV status of the mother and child were obtained during the interview or review of the medical records. If the status of the child was unknown and the parent gave consent, then a dried blood spot was collected for anonymised HIV testing.

\section{Laboratory screening}

A stool specimen was collected for enteric pathogen detection. Nucleic acids were extracted from 10\% stool suspensions using the QIAamp ${ }^{\circledR}$ Viral RNA Mini Kit (Qiagen Inc., Valencia, CA) according to the manufacturer's instructions and eluted in $60 \mu \mathrm{l}$ Rase-free water. Briefly, $10 \mu \mathrm{l}$ extracted RNA was reverse transcribed with random primers using the Transcriptor First Strand cDNA Synthesis Kit (Roche, Mannheim, Germany) according to the manufacturers' instructions. Samples were screened for NoV GI and GII in monoplex reactions using $5 \mu \mathrm{l}$ cDNA, LightCycler ${ }^{\circledR} 480$ Probe Master Kit (Roche) and primers and probes from published methods [18-20]. Screening of other enteric viruses, bacteria and parasites has been described elsewhere [21, 22].

A fully screened specimen was defined as a specimen where all viral, bacterial and parasite testing had been performed $(n=1354)$.

Dried blood spots were screened using the AmpliPrep ${ }^{\mathbb{R}}$ (Roche) for automated nucleic acid extraction and COBAS $^{\circledR}$ TaqMan $^{\circledR}$ (Roche) for automated real-time detection of HIV-1.

\section{Data analysis}

NoV detection rate was calculated using data from years with 12 months of surveillance (2010-2013). Demographic data, clinical characteristics and environmental features associated with $\mathrm{NoV}$ detection were compared in patients with or without NoV, genogroup I (GI) or II (GII) using Stata12 (StataCorp LP, College Station TX). In addition, the characteristics of HIV-infected and HIV-uninfected children were compared in NoV-positive cases. NoV-negative cases were defined as cases where neither NoV GI nor GII strains were detected. Mixed infections were defined as detection of NoV GI or GII strains with any of the following pathogens: RV, human adenovirus (any group), astrovirus, sapovirus ( $\mathrm{SaV}$ ), bocavirus, bacteria (Campylobacter spp., diffusely adherent Escherichia coli, enteroaggregative E. coli, enteroinvasive E. coli, enteropathogenic E. coli, enterotoxigenic E. coli, Salmonella spp. and Shigella spp.) and parasites (Ascaris lumbricoides, Cryptosporidium spp., Entamoeba coli, Giardia lamblia and Isospora belli).

The $\chi^{2}$ tests were used to determine statistical significance $(P \leqslant 0.05)$ of categorical data, while $t$ tests with unequal variance and Welch's approximation were used for means. A Wilcoxon rank-sum test was used to assess differences between medians. Univariate analysis and stepwise multivariable logistic regression analysis was performed to identify environmental features associated with $\mathrm{NoV}$ detection in patients with diarrhoea and a separate analysis restricted to NoV-positive cases compared the characteristics of HIV-infected and HIV-uninfected children. Variables that did not yield statistically significant $P$-values on univariate analysis were not included in the multivariable models or reported in the tables.

\section{RESULTS}

Between 20 April 2009 and 31 December 2013, 3103 children $<5$ years provided stool samples and were included in the surveillance study. All samples were screened for NoV GI and GII, SaV, human adenovirus, astrovirus and bocavirus. In addition, specimens were screened for RV $(99 \cdot 7 \% ; 3093 / 3103)$, bacteria $(88.6 \% ; 2748 / 3103)$ and parasites $(48 \cdot 5 \% ; 1508 / 3103)$.

Overall, $2 \cdot 6 \%(79 / 3019)$ of children admitted to the sentinel hospitals for the treatment of diarrhoea died over the study period. While $10 \%(8 / 79)$ of these children had NoV detected, only two children had NoV GII strains as the sole pathogen detected. Of the eight children that died, four were HIV infected.

\section{NoV detection}

Between 2009 and 2013, NoV was detected in 452/ $3103(14.6 \%)$ of specimens. The detection rate when only years with 12 months of data were included was 16\% (396/2468; 2010-2013). NoV GII strains were detected in $13.4 \%(330 / 2468 ; 95 \%$ CI $12 \cdot 1-$ $14 \cdot 8)$ of specimens and NoV GI in 3.4\% (84/2468; 95\% CI 2.4-3.6) of specimens between 2010 and 2013. An additional 47 GII and 10 GI cases were detected between April and December 2009, resulting in 377 NoV GII and 94 NoV GI cases available for analysis (Supplementary Tables 1 and 2). In fully screened NoV-positive specimens, NoV GII strains were detected as the sole pathogen in $35.4 \%$ (61/172) 
and GI in $23.4 \%(11 / 47)$ of cases. Mixed GII and GI NoV detected in $19(0.6 \% ; 19 / 3103)$ diarrhoea cases.

The median cycle threshold $\left(C_{\mathrm{t}}\right)$ values in these specimens were compared in single and mixed pathogen NoV cases. The median $C_{\mathrm{t}}$ values of single $\left(C_{\mathrm{t}}=\right.$ 27·1; IQR 18.6-31·1) pathogen GII cases were significantly lower than mixed $\left(C_{\mathrm{t}}=30 \cdot 6\right.$; IQR $\left.22 \cdot 0-33 \cdot 4\right)$ pathogen NoV GII cases $(P=0 \cdot 005)$. Similarly, the median $C_{\mathrm{t}}$ values of single pathogen GI cases were significantly lower than mixed pathogen NoV GI cases $\left(n=47\right.$; single: $C_{\mathrm{t}}=27 \cdot 2$ (IQR 21.0-29.0); mixed: $C_{\mathrm{t}}=32 \cdot 0$ (IQR 28.0-33.9); $P=0 \cdot 008$ ).

\section{NoV epidemiology}

NoV detection was higher in children 0-6 months and 7-12 months compared with children older than 24 months (0-6 months adjusted odds ratio (aOR) $2 \cdot 2$; $95 \%$ CI $1 \cdot 4-3 \cdot 6 ; \quad P=0 \cdot 001 ; 7-12$ months aOR $2 \cdot 2$ $95 \%$ CI $1 \cdot 3-3 \cdot 5 ; P=0 \cdot 002$; Table 1 ). A similar age trend was noted in NoV GII cases (Supplementary Table 1). Unlike NoV GII, the odds of NoV GI detection were higher in the second year of life (19-24 months; aOR 2.3; 95\% CI $1 \cdot 2-4.5 ; \quad P=0.011$; Supplementary Table 2) compared with the 0-6month age group (Supplementary Table 2).

NoV strains were frequently detected in 2010 and 2011 compared with 2013 (2010; aOR 2.2 (95\% CI $1 \cdot 5-3 \cdot 1) ; P<0 \cdot 001$ and 2011 ; aOR $2 \cdot 1(95 \%$ CI $1 \cdot 4$ $3.0) ; P<0.001$; Table 1). This result was echoed in the individual analyses of GII and GI strains although the result was not statistically significant in multivariable analysis of GI (Supplementary Tables 1 and 2). The NoV detection rate by sentinel site was similar ranging from $10 \cdot 0 \%$ in $\mathrm{MPH}$ to $16 \cdot 1 \%$ in $\mathrm{EDH}$ over the study period (Table 1).

NoV GII were frequently detected from September to December each year (Fig. 1). The percentagepositive NoV GII during this period was $20.5 \%$ $(163 / 797)$ compared with the rest of the year $(9 \cdot 3 \%$; $214 / 2306 ; P<0 \cdot 001)$. The monthly detection rate of NoV GI strains in SA was less pronounced than NoV GII although the average detection in late summer (January-April) was 4.3\% (43/1009) compared with $2.4 \%$ for the rest of the year (May-December; 51/2094; $P=0 \cdot 01$; Fig. 1).

\section{Clinical characteristics of NoV infections}

Vomiting was more frequently identified in NoVpositive compared with NoV-negative children (OR
$1 \cdot 3 ; \quad 95 \%$ CI $1 \cdot 1-1 \cdot 7 ; \quad P=0 \cdot 011 ;$ Table 1$)$. NoV GII-positive patients were more likely to report five or more vomiting episodes per day (OR 1.9; 95\% CI $1 \cdot 2$ $2 \cdot 8 ; P=0.003)$ and vomiting duration of up to 2 days (aOR $1 \cdot 8 ; 95 \%$ CI $1 \cdot 2-2 \cdot 7 ; P=0.005$ ) compared with NoV-negative patients (Supplementary Table 1). However, NoV-positive cases were less likely to be dehydrated when compared with NoV-negative cases (aOR $0 \cdot 7-0 \cdot 5 ; 95 \%$ CI $0 \cdot 3-0 \cdot 9 ; P=0 \cdot 009$; Table 1). Children with NoV detected were admitted to hospital for a median of 2 days (IQR 1-4) compared with 3 days (IQR 1-6) in children without NoV detected $(P<$ $0 \cdot 001)$. Low weight at birth (aOR 0.6; 95\% CI 0.4-0.9; $P=0.017$ ) compared with normal weight at birth was significantly less common in NoV GII-positive compared with NoV GII-negative patients (Supplementary Table 1).

\section{Environmental features associated with NoV detection}

Informal housing compared with brick houses (aOR $0 \cdot 7 ; 95 \%$ CI $0.5-1 \cdot 0 ; P=0.021$; Table 1 ) was significantly less common in NoV-positive than NoVnegative cases. Water from outdoor taps and boreholes (aOR 0.7; 95\% CI 0.5-1.0; $P=0 \cdot 045$ ) compared with indoor taps and mixed infections with RV (aOR $0 \cdot 6$; 95\% CI $0.4-0.8 ; P=0.002)$ and human adenovirus (aOR $0 \cdot 3 ; 95 \%$ CI $0 \cdot 2-0 \cdot 6 ; P<0 \cdot 001$ ) were all significantly less common in NoV GII-positive than NoV GII-negative patients (Supplementary Table 1). No statistically significant environmental or behavioural features were associated with NoV GI detection (Supplementary Table 2).

Compared with NoV-negative cases, mixed pathogen infections were frequently associated with $\mathrm{NoV}$ detection (NoV GII + one other pathogen (OR 1.8; $95 \%$ CI $1 \cdot 3-2 \cdot 6 ; \quad P=0 \cdot 001)$ and $\mathrm{NoV}$ GII $+\geqslant 2$ other pathogens (OR 5.2; 95\% CI 3.5-7.7; $P<$ $0 \cdot 001)$ compared with NoV only; Table 1). These results were also significant when NoVs were stratified according to genogroup (Supplementary Tables 1 and 2).

The odds of detecting NoV GI strains increased 2.0 times when NoV GII strains were present $(95 \%$ CI $1 \cdot 2-3 \cdot 3)$ and $2 \cdot 0$ times when $\mathrm{SaV}$ was present $(95 \%$ CI 1·1-3.7; Supplementary Table 2) compared with single NoV GI cases. The association between NoV $\mathrm{GI}$ and $\mathrm{SaV}$ was maintained when adjusted for month of collection (aOR $1.9(95 \%$ CI $1 \cdot 0-3 \cdot 5) ; P=$ 0.041). Similarly, the NoV GI/NoV GII association also maintained statistical significance when adjusted 
Table 1. Univariate and multivariable analysis of demographic data, clinical characteristics and environmental features associated with NoV detection $(n=452)$

\begin{tabular}{|c|c|c|c|c|c|}
\hline \multirow[b]{2}{*}{ Parameter } & \multirow{2}{*}{$\begin{array}{l}\text { NoV detection } \\
n / N(\%)\end{array}$} & \multicolumn{2}{|c|}{ Univariate analysis } & \multicolumn{2}{|c|}{ Multivariable analysis } \\
\hline & & OR $(95 \% \mathrm{CI})$ & $P$-value & OR $(95 \% \mathrm{CI})$ & $P$-value \\
\hline \multicolumn{6}{|l|}{ Demographic characteristics } \\
\hline \multicolumn{6}{|l|}{ Age (in months) } \\
\hline$>24$ & $29 / 302(9 \cdot 6)$ & Ref & & Ref & \\
\hline $19-24$ & $38 / 232(16 \cdot 4)$ & $1 \cdot 8(1 \cdot 1-3 \cdot 1)$ & $0 \cdot 020$ & $1 \cdot 8(1 \cdot 0-3 \cdot 3)$ & $0 \cdot 051$ \\
\hline $13-18$ & $56 / 459(12 \cdot 2)$ & $1 \cdot 3(0 \cdot 8-2 \cdot 1)$ & $0 \cdot 267$ & $1 \cdot 7(1 \cdot 0-2 \cdot 9)$ & $0 \cdot 063$ \\
\hline $7-12$ & $149 / 915(16 \cdot 3)$ & $1 \cdot 8(1 \cdot 2-2 \cdot 8)$ & $0 \cdot 005$ & $2 \cdot 2(1 \cdot 3-3 \cdot 5)$ & $0 \cdot 002$ \\
\hline $0-6$ & $179 / 1191(15 \cdot 0)$ & $1 \cdot 7(1 \cdot 1-2 \cdot 5)$ & $0 \cdot 016$ & $2 \cdot 2(1 \cdot 4-3 \cdot 6)$ & $0 \cdot 001$ \\
\hline \multicolumn{6}{|l|}{ Year } \\
\hline 2013 & $56 / 537(10 \cdot 4)$ & Ref & & Ref & \\
\hline 2012 & $56 / 459(12 \cdot 2)$ & $1 \cdot 2(0 \cdot 8-1 \cdot 8)$ & $0 \cdot 378$ & $1 \cdot 2(0 \cdot 8-1 \cdot 9)$ & $0 \cdot 343$ \\
\hline 2011 & $101 / 553(18 \cdot 3)$ & $1 \cdot 9(1 \cdot 4-2 \cdot 7)$ & $<0 \cdot 001$ & $2 \cdot 1(1 \cdot 4-3 \cdot 0)$ & $<0.001$ \\
\hline 2010 & $183 / 919(19 \cdot 9)$ & $2 \cdot 1(1 \cdot 5-2 \cdot 9)$ & $<0 \cdot 001$ & $2 \cdot 2(1 \cdot 5-3 \cdot 1)$ & $<0.001$ \\
\hline \multicolumn{6}{|l|}{ Sentinel site } \\
\hline $\mathrm{CHBH}$ & $274 / 1764(15 \cdot 5)$ & Ref & & & \\
\hline EDH & $50 / 310(16 \cdot 1)$ & $1 \cdot 0(0 \cdot 8-1 \cdot 5)$ & $0 \cdot 790$ & & \\
\hline MPH & $36 / 359(10 \cdot 0)$ & $0.6(0 \cdot 4-0.9)$ & $0 \cdot 008$ & & \\
\hline MKH & $92 / 670(13 \cdot 7)$ & $0.9(0 \cdot 7-1 \cdot 1)$ & $0 \cdot 267$ & & \\
\hline \multicolumn{6}{|l|}{ Clinical characteristics } \\
\hline \multicolumn{6}{|l|}{ Diarrhoea duration (in days) } \\
\hline$\geqslant 6$ & $34 / 203(16 \cdot 8)$ & Ref & & & \\
\hline 5 & $31 / 260(11 \cdot 9)$ & $0 \cdot 6(0 \cdot 4-1 \cdot 1)$ & $0 \cdot 140$ & & \\
\hline $1-4$ & $375 / 2571(14 \cdot 6)$ & $0 \cdot 8(0 \cdot 6-1 \cdot 2)$ & $0 \cdot 403$ & & \\
\hline \multicolumn{6}{|l|}{ Vomiting } \\
\hline No & $119 / 981(12 \cdot 1)$ & Ref & & \multicolumn{2}{|c|}{ Not included in model } \\
\hline Yes & $324 / 2079(15 \cdot 6)$ & $1 \cdot 3(1 \cdot 1-1 \cdot 7)$ & $0 \cdot 011$ & & \\
\hline \multicolumn{6}{|l|}{ Vomiting duration (in days) } \\
\hline 0 & $119 / 981(12 \cdot 1)$ & Ref & & Ref & \\
\hline 1 & $67 / 437(15 \cdot 3)$ & $1 \cdot 3(0 \cdot 9-1 \cdot 8)$ & $0 \cdot 100$ & $1 \cdot 3(0 \cdot 9-1 \cdot 8)$ & $0 \cdot 228$ \\
\hline 2 & $111 / 655(17 \cdot 0)$ & $1 \cdot 4(1 \cdot 1-2 \cdot 0)$ & $0 \cdot 006$ & $1 \cdot 4(1 \cdot 0-1 \cdot 9)$ & $0 \cdot 041$ \\
\hline$\geqslant 3$ & $143 / 971(14 \cdot 7)$ & $1 \cdot 3(1 \cdot 0-1 \cdot 6)$ & $0 \cdot 093$ & $1 \cdot 1(0 \cdot 8-1 \cdot 5)$ & $0 \cdot 540$ \\
\hline \multicolumn{6}{|c|}{ Maximum number of vomits per $24 \mathrm{~h}$} \\
\hline 0 & $119 / 981(12 \cdot 1)$ & Ref & & \multirow{4}{*}{\multicolumn{2}{|c|}{ Not included in model }} \\
\hline 1 & $39 / 337(11 \cdot 5)$ & $0.9(0 \cdot 6-1 \cdot 4)$ & $0 \cdot 786$ & & \\
\hline $2-4$ & $221 / 1410(15 \cdot 7)$ & $1 \cdot 3(1 \cdot 1-1 \cdot 7)$ & $0 \cdot 015$ & & \\
\hline$\geqslant 5$ & $45 / 233(19 \cdot 3)$ & $1 \cdot 7(1 \cdot 2-2 \cdot 5)$ & $0 \cdot 004$ & & \\
\hline \multicolumn{6}{|l|}{ Admission temperature $\left({ }^{\circ} \mathrm{C}\right)$} \\
\hline$\leqslant 37 \cdot 0$ & $151 / 942(16 \cdot 0)$ & Ref & & & \\
\hline $37 \cdot 1-38 \cdot 4$ & $90 / 588(15 \cdot 3)$ & $0 \cdot 9(0 \cdot 7-1 \cdot 3)$ & $0 \cdot 705$ & & \\
\hline $38 \cdot 5-38 \cdot 9$ & $18 / 82(22 \cdot 0)$ & $1 \cdot 5(0 \cdot 8-2 \cdot 6)$ & $0 \cdot 168$ & & \\
\hline$\geqslant 39 \cdot 0$ & $19 / 92(20 \cdot 7)$ & $1 \cdot 4(0 \cdot 8-2 \cdot 3)$ & $0 \cdot 255$ & & \\
\hline \multicolumn{6}{|c|}{ Dehydration (as assessed by a clinician) } \\
\hline None & $84 / 414(20 \cdot 1)$ & Ref & & Ref & \\
\hline $1-5 \%$ (mild) & $244 / 1658(14 \cdot 7)$ & $0 \cdot 7(0 \cdot 5-0 \cdot 9)$ & $0 \cdot 006$ & $0 \cdot 7(0 \cdot 5-0 \cdot 9)$ & $0 \cdot 009$ \\
\hline$\geqslant 6 \%$ (moderate/severe $)$ & $79 / 649(12 \cdot 2)$ & $0 \cdot 5(0 \cdot 4-0 \cdot 8)$ & $<0.001$ & $0 \cdot 5(0 \cdot 3-0 \cdot 7)$ & $0 \cdot 001$ \\
\hline \multicolumn{6}{|l|}{ Child's HIV status } \\
\hline Negative & $374 / 2467(15 \cdot 2)$ & Ref & & & \\
\hline Positive & $44 / 357(12 \cdot 3)$ & $0 \cdot 8(0 \cdot 6-1 \cdot 1)$ & $0 \cdot 159$ & & \\
\hline \multicolumn{6}{|l|}{ Child's HIV exposure } \\
\hline Uninfected, unexposed & $260 / 1693(15 \cdot 4)$ & Ref & & & \\
\hline Uninfected, exposed & $128 / 924(13 \cdot 9)$ & $0 \cdot 9(0 \cdot 7-1 \cdot 1)$ & $0 \cdot 301$ & & \\
\hline Infected & $44 / 357(12 \cdot 3)$ & $0 \cdot 8(0 \cdot 6-1 \cdot 1)$ & $0 \cdot 144$ & & \\
\hline Number of days admitted & & & & & \\
\hline$\geqslant 3$ & $190 / 1559(12 \cdot 2)$ & Ref & & & \\
\hline
\end{tabular}


Table 1 (cont.)

\begin{tabular}{|c|c|c|c|c|c|}
\hline \multirow[b]{2}{*}{ Parameter } & \multirow{2}{*}{$\begin{array}{l}\text { NoV detection } \\
n / N(\%)\end{array}$} & \multicolumn{2}{|c|}{ Univariate analysis } & \multicolumn{2}{|c|}{ Multivariable analysis } \\
\hline & & OR $(95 \% \mathrm{CI})$ & $P$-value & OR $(95 \% \mathrm{CI})$ & $P$-value \\
\hline$\leqslant 2$ & $248 / 1465(16 \cdot 9)$ & $1 \cdot 5(1 \cdot 2-1 \cdot 8)$ & $<0 \cdot 001$ & $1 \cdot 3(1 \cdot 0-1 \cdot 7)$ & $0 \cdot 023$ \\
\hline \multicolumn{6}{|l|}{ Child's birth weight } \\
\hline$\geqslant 2.5 \mathrm{~kg}$ & $263 / 1548(17 \cdot 0)$ & Ref & & & \\
\hline$<2.5 \mathrm{~kg}$ & $36 / 303(11.9)$ & $0 \cdot 7(0 \cdot 5-1 \cdot 0)$ & $0 \cdot 028$ & & \\
\hline \multicolumn{6}{|c|}{$\begin{array}{l}\text { Environmental features associated with } \mathrm{NoV} \\
\text { detection }\end{array}$} \\
\hline \multicolumn{6}{|c|}{ Housing material } \\
\hline Brick & $355 / 2316(15 \cdot 3)$ & Ref & & & \\
\hline Informal & $85 / 736(11 \cdot 6)$ & $0 \cdot 7(0 \cdot 6-0 \cdot 9)$ & $0 \cdot 011$ & $0 \cdot 7(0 \cdot 5-1 \cdot 0)$ & $0 \cdot 021$ \\
\hline \multicolumn{6}{|l|}{ Water source } \\
\hline In-door tap & $225 / 1414(15 \cdot 9)$ & Ref & & & \\
\hline Other & $217 / 1637(13 \cdot 3)$ & $0 \cdot 8(0 \cdot 7-1 \cdot 0)$ & $0 \cdot 038$ & & \\
\hline \multicolumn{6}{|l|}{ Sanitation type } \\
\hline Flush toilet & $253 / 1591(15 \cdot 9)$ & Ref & & & \\
\hline Other & $190 / 1462(13 \cdot 0)$ & $0 \cdot 8(0 \cdot 6-1 \cdot 0)$ & $0 \cdot 023$ & & \\
\hline \multicolumn{6}{|c|}{ NoV mixed pathogen infections } \\
\hline NoV only & $72 / 592(12 \cdot 2)$ & Ref & & \multirow{7}{*}{\multicolumn{2}{|c|}{ Not included in model }} \\
\hline $\mathrm{NoV}+1$ & $75 / 370(20 \cdot 3)$ & $1 \cdot 8(1 \cdot 3-2 \cdot 6)$ & $0 \cdot 001$ & & \\
\hline $\mathrm{NoV}+2$ or more & $68 / 163(41 \cdot 7)$ & $5 \cdot 2(3 \cdot 5-7 \cdot 7)$ & $<0.001$ & & \\
\hline $\mathrm{NoV}+$ rotavirus & $100 / 895(11 \cdot 2)$ & $0.7(0 \cdot 5-0.8)$ & 0.001 & & \\
\hline $\mathrm{NoV}+$ adenovirus & $71 / 579(9 \cdot 3)$ & $0 \cdot 8(0 \cdot 6-1 \cdot 0)$ & $0 \cdot 082$ & & \\
\hline $\mathrm{NoV}+$ astrovirus & $45 / 214(21 \cdot 0)$ & $1 \cdot 6(1 \cdot 1-2 \cdot 3)$ & $0 \cdot 006$ & & \\
\hline $\mathrm{NoV}+$ parasites & $18 / 190(9 \cdot 5)$ & $0 \cdot 6(0 \cdot 4-1 \cdot 0)$ & $0 \cdot 039$ & & \\
\hline
\end{tabular}

CHBH, Chris Hani Baragwanath Hospital; EDH, Edendale Hospital; MPH, Mapulaneng Hospital; MKH, Matikwane Hospital; HIV, human immunodeficiency virus.

Only variables with $P$-values $<0 \cdot 2$ in the univariate analysis were reported in the table and included in the multivariable model.

for month of collection (aOR $1 \cdot 9(95 \%$ CI $1 \cdot 1-3 \cdot 3)$; $P=0 \cdot 017)$.

\section{NoV infection in HIV-infected and -uninfected children}

The HIV status was available for $91 \%$ (2824/3103) of the children enrolled and for 96\% (2974/3103) of mothers. The HIV status was missing in 7\% (26/377) of the NoV GII cases and 9\% (8/94) of the NoV GI cases. Mixed GII and GI infections were noted in 19 cases with 418 cases available for analysis.

The detection of single ( $11 \%$ vs. $10 \%)$ pathogen $\mathrm{NoV}$ GII cases was similar in HIV-infected and HIVuninfected children $(P=1 \cdot 0)$. The detection of mixed pathogen NoV GII cases was lower in HIV-infected (9\%; 26/278) compared with HIV-uninfected children (14\%; 267/1954; $P=0 \cdot 046)$. There were no differences in the median NoV GII $C_{\mathrm{t}}$ values in HIV-infected $\left(C_{\mathrm{t}}=27.9\right.$; IQR 21.4-32.0) compared with HIVuninfected children $\left(C_{\mathrm{t}}=26 \cdot 1\right.$; IQR $19 \cdot 7-32 \cdot 0 ; P=$ $0 \cdot 45)$.
The detection of single pathogen NoV GI infections was higher in HIV-infected $(9 \%$; 6/67) compared with HIV-uninfected $(1 \% ; 4 / 410 ; P<0.001)$ children while the detection of mixed pathogen NoV GI infections was lower in HIV-infected $(2 \%$; 5/257) compared with HIV-uninfected children (4\%; 71/1758; $P=0 \cdot 115)$. Univariate analysis indicated that the odds of detecting single pathogen NoV GI in HIV-infected children were $10 \cdot 0$ times greater $(95 \%$ CI $2 \cdot 7-36 \cdot 4)$ compared with HIV-uninfected children $(P<0 \cdot 001)$. In addition, the median NoV GI $\mathrm{Ct}$ values in HIV-infected children were significantly lower $\left(C_{\mathrm{t}}=28 \cdot 0\right.$; IQR $\left.24 \cdot 4-31 \cdot 1\right)$ compared with HIV-uninfected children $\left(C_{\mathrm{t}}=32 \cdot 0\right.$; IQR 26.9-34.2; $P=0 \cdot 05$ ) on univariate analysis.

Amongst children with $\mathrm{NoV}$ detected, HIV-infected children displayed a higher case fatality rate $(9 \cdot 3 \% \mathrm{vs}$. $1.0 \%$; aOR $6.9 ; 95 \%$ CI $1.5-31.9 ; P=0.013)$ and longer hospitalizations ( $\geqslant 3$ days $74 \cdot 4 \%$ vs. $39 \cdot 9 \%$; aOR $4 \cdot 3 ; 95 \%$ CI $2 \cdot 1-8 \cdot 9 ; P<0 \cdot 001$; Table 2) than HIV-uninfected children on multivariable analysis. In addition, HIV-infected children with $\mathrm{NoV}$ had 


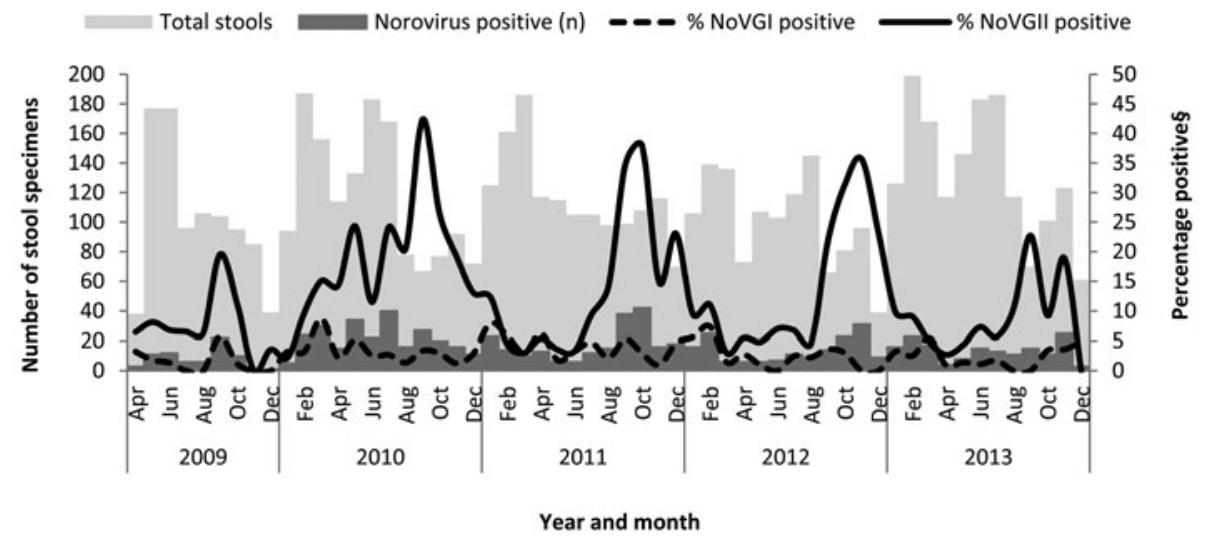

${ }^{5}$ Percentage positive NoV GII or GI = number of NoV GII or GI positive cases per month/ total number of specimens screened per month*100; secondary axis scale drawn to $50 \%$.

Fig. 1. Total stools tested, number of NoV-positive specimens and the percentage positive for NoV GI and GII by month

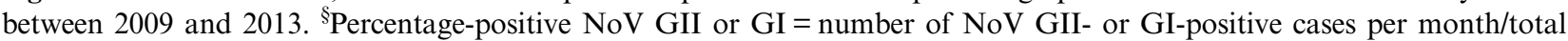
number of specimens screened per month $\times 100$; secondary axis scale drawn to $50 \%$.

$2 \cdot 4$ times greater odds than HIV-uninfected children of experiencing diarrhoea duration of 3 days or more compared with 2 days or less $(95 \%$ CI $1 \cdot 2-4 \cdot 9$; $P=0 \cdot 018 ;$ Table 2).

\section{DISCUSSION}

The study provides epidemiological features of NoV infection in hospitalized children $<5$ years in a setting with high HIV prevalence. The study demonstrated the importance of NoV in hospitalized children aged $<2$ years and identified diarrhoea and vomiting with limited dehydration as prominent clinical characteristics in NoV cases. Amongst NoV-infected children, HIV-infection was associated with prolonged hospitalization and increased mortality. It is the first study of its kind in SA that investigates NoV detection in HIV-infected children and NoV diarrhoea post rotavirus vaccine introduction. The study also spans more than 12 months, includes more than one hospital and geographic area and assesses data on clinical characteristics and the environmental features associated with NoV detection in children $<5$ years.

An earlier study in SA in 2008 detected NoV in 14\% of hospitalized children $<2$ years [23]. Previous studies in Africa have also identified $\mathrm{NoV}$ in an average of $13.5 \%$ of diarrhoea cases [9]. Similarly, a worldwide systematic review of studies conducted between 1990 and 2008 estimated that $\mathrm{NoV}$ was responsible for $12 \%$ of severe diarrhoea cases in children $<5$ years [3]. These results are comparable with the current study findings with NoV detection (GI and GII strains) at $15 \%$ in hospitalized South African children.

NoV has often been described as 'winter vomiting disease' due to the winter seasonality and occurrence of vomiting symptoms [6]. Globally, NoV infections seem to be more common during the winter months [24, 25]. However, reports from Spain in 2001 and the UK in 2002 show that summer peaks are occasionally present [26, 27]. In SA, NoVs were detected throughout the year with detection increasing during the warmer spring and summer months in SA (between September and November). The results obtained in this study are similar to those obtained by Mans et al. at two hospitals in Gauteng Province in 2008 [23].

During 2010, NoV GII strains were detected at levels above $20 \%$ for 5 months (May and JulyOctober), with an uncharacteristic autumn/winter predominance. Whether or not this increase was related to increased tourist activity and the introduction of new GII and GI strains during the Soccer World Cup in SA in June and July of 2010 or the decrease in rotavirus due to the introduction of the rotavirus vaccine in 2009 is unknown. Since the New Orleans 2009 GII.4 variant was first detected in SA in 2008 and was regularly detected from April 2009 to December 2012, the increase is unlikely to be related to changes in the dominant GII.4 variant [28].

NoV GII strains identified in a 2008 study in Pretoria indicated that eight GII (GII.1, GII.4, GII.6, GII.7, GII.10, GII.13, GII.14, GII.16) strains 
Table 2. Univariate and multivariable analysis of demographic data, clinical characteristics and environmental features associated with NoV detection in HIV-infected and HIV-uninfected children $(n=418)$

\begin{tabular}{|c|c|c|c|c|c|c|}
\hline \multirow[b]{2}{*}{ Parameter } & \multirow{2}{*}{$\begin{array}{l}\text { HIV-infected } \\
\mathrm{n} / \mathrm{N}(\%)\end{array}$} & \multirow{2}{*}{$\begin{array}{l}\text { HIV-uninfected } \\
\mathrm{n} / \mathrm{N}(\%)\end{array}$} & \multicolumn{2}{|c|}{ Univariate analysis } & \multicolumn{2}{|c|}{ Multivariable analysis } \\
\hline & & & OR $(95 \% \mathrm{CI})$ & $P$-value & $\mathrm{aOR}(95 \% \mathrm{CI})$ & $P$-value \\
\hline \multicolumn{7}{|l|}{ Gender } \\
\hline Male & $20 / 44(45 \cdot 5)$ & $218 / 374(58 \cdot 3)$ & Ref & & & \\
\hline Female & $24 / 44(54 \cdot 5)$ & $156 / 374(41 \cdot 7)$ & $1 \cdot 7(0 \cdot 9-3 \cdot 1)$ & $0 \cdot 107$ & & \\
\hline \multicolumn{7}{|l|}{ Sentinel site } \\
\hline $\mathrm{CHBH}$ & $16 / 44(36 \cdot 4)$ & $232 / 374(62 \cdot 0)$ & Ref & & & \\
\hline EDH & $5 / 44(11 \cdot 4)$ & $42 / 374(11 \cdot 2)$ & $1 \cdot 7(0 \cdot 6-5 \cdot 0)$ & $0 \cdot 311$ & & \\
\hline MKH & $19 / 44(43 \cdot 2)$ & $69 / 374(18 \cdot 5)$ & $4 \cdot 0(1 \cdot 9-8 \cdot 2)$ & $<0 \cdot 001$ & & \\
\hline MPH & $4 / 44(9 \cdot 1)$ & $31 / 374(8 \cdot 3)$ & $1.9(0 \cdot 6-6 \cdot 0)$ & $0 \cdot 289$ & & \\
\hline \multicolumn{7}{|l|}{ Year } \\
\hline 2013 & $3 / 44(6 \cdot 8)$ & $51 / 374(13 \cdot 6)$ & Ref & & & \\
\hline 2012 & $6 / 44(13 \cdot 6)$ & $46 / 374(13 \cdot 6)$ & $2 \cdot 2(0 \cdot 5-9 \cdot 4)$ & $0 \cdot 279$ & & \\
\hline 2011 & $7 / 44(15 \cdot 9)$ & $92 / 374(24 \cdot 6)$ & $1 \cdot 3(0 \cdot 3-5 \cdot 2)$ & 0.718 & & \\
\hline 2010 & $25 / 44(56 \cdot 8)$ & $140 / 374(37 \cdot 4)$ & $3 \cdot 0(0 \cdot 9-10 \cdot 5)$ & 0.079 & & \\
\hline 2009 & $3 / 44(6 \cdot 8)$ & $45 / 374(12 \cdot 0)$ & $1 \cdot 1(0 \cdot 2-5 \cdot 9)$ & $0 \cdot 882$ & & \\
\hline \multicolumn{7}{|l|}{ Diarrhoea duration (in days) } \\
\hline$\leqslant 2$ & $12 / 44(27 \cdot 3)$ & $170 / 369(46 \cdot 1)$ & Ref & & Ref & \\
\hline$\geqslant 3$ & $32 / 44(72 \cdot 7)$ & $199 / 369(53 \cdot 9)$ & $2 \cdot 3(1 \cdot 1-4 \cdot 6)$ & $0 \cdot 020$ & $2 \cdot 4(1 \cdot 2-4 \cdot 9)$ & $0 \cdot 018$ \\
\hline \multicolumn{7}{|l|}{ Vomiting duration (in days) } \\
\hline$\leqslant 2$ & $25 / 44(56 \cdot 8)$ & $261 / 372(70 \cdot 2)$ & Ref & & & \\
\hline$\geqslant 3$ & $19 / 44(43 \cdot 2)$ & $111 / 372(29 \cdot 8)$ & $1 \cdot 8(0 \cdot 9-3 \cdot 4)$ & $0 \cdot 074$ & & \\
\hline \multicolumn{7}{|c|}{ Dehydration (as assessed by a clinician) } \\
\hline None-mild (1-5\%) & $27 / 40(67 \cdot 5)$ & 280/344 (81·4) & Ref & & & \\
\hline$\geqslant 6 \%$ (moderate/severe) & $13 / 40(32 \cdot 5)$ & $64 / 344(18 \cdot 6)$ & $2 \cdot 1(1 \cdot 0-4 \cdot 3)$ & $0 \cdot 041$ & & \\
\hline \multicolumn{7}{|l|}{ Bloody stools } \\
\hline No & $36 / 44(81 \cdot 8)$ & $333 / 370(90 \cdot 0)$ & Ref & & & \\
\hline Yes & $8 / 44(18 \cdot 2)$ & $37 / 370(10 \cdot 0)$ & $2 \cdot 0(0 \cdot 9-4 \cdot 6)$ & $0 \cdot 105$ & & \\
\hline \multicolumn{7}{|c|}{ Number of days admitted to hospital } \\
\hline$\leqslant 2$ & $11 / 43(25 \cdot 6)$ & $221 / 368(60 \cdot 1)$ & Ref & & Ref & \\
\hline$\geqslant 3$ & $32 / 43(74 \cdot 4)$ & $147 / 368(39 \cdot 9)$ & $4 \cdot 4(2 \cdot 1-9 \cdot 0)$ & $<0 \cdot 001$ & $4 \cdot 3(2 \cdot 1-8 \cdot 9)$ & $<0 \cdot 001$ \\
\hline \multicolumn{7}{|c|}{ Number of inhabitants living in house } \\
\hline$\leqslant 6$ & $31 / 35(88 \cdot 6)$ & $268 / 283(94 \cdot 7)$ & Ref & & & \\
\hline$\geqslant 7$ & $4 / 35(11 \cdot 4)$ & $15 / 283(5 \cdot 3)$ & $2 \cdot 3(0 \cdot 7-7 \cdot 4)$ & $0 \cdot 160$ & & \\
\hline \multicolumn{7}{|l|}{ Sanitation type } \\
\hline Flush toilet & $14 / 44(31 \cdot 8)$ & $222 / 372(59 \cdot 7)$ & Ref & & & \\
\hline Other & $30 / 44(68 \cdot 2)$ & $150 / 372(40 \cdot 3)$ & $3 \cdot 2(1 \cdot 6-6 \cdot 2)$ & $0 \cdot 001$ & & \\
\hline \multicolumn{7}{|l|}{ Outcome } \\
\hline Discharged & $39 / 43(90 \cdot 7)$ & $365 / 369(98 \cdot 9)$ & Ref & & Ref & \\
\hline Died & $4 / 43(9 \cdot 3)$ & $4 / 369(1 \cdot 0)$ & $9 \cdot 4(2 \cdot 3-38 \cdot 9)$ & $0 \cdot 002$ & $6 \cdot 9(1 \cdot 5-31 \cdot 9)$ & $0 \cdot 013$ \\
\hline \multicolumn{7}{|c|}{ NoV GII mixed pathogen infections } \\
\hline NoV GII +rotavirus & $5 / 44(11 \cdot 4)$ & $89 / 372(23 \cdot 9)$ & $0 \cdot 4(0 \cdot 2-1 \cdot 1)$ & $0 \cdot 067$ & & \\
\hline
\end{tabular}

CHBH, Chris Hani Baragwanath Hospital; EDH, Edendale Hospital; MKH, Matikwane Hospital; MPH, Mapulaneng Hospital; HIV, human immunodeficiency virus.

Only variables with $P$-values $<0 \cdot 2$ in the univariate analysis were reported in the table and included in the multivariable model.

were circulating [23]. While genotyping data from 2010 revealed that GII.2, GII.3, GII.4, GII.12 and GII.17 strains were circulating [29], only limited typing was performed on the 2010 GII strains (39/216; $18 \%$ ). Therefore, with the current data available, the introduction of new virus genotypes or variants as the cause of the increased detection of NoV GII and GI infections in 2010 could not be established. Continued surveillance and genotyping of NoV strains is recommended to identify the introduction of new 
variants or types in the South African paediatric population.

In agreement with recent NoV data [6, 30], vomiting was a significant symptom associated with NoV detection in South African children hospitalized for diarrhoea. However, children with NoV were not dehydrated and admitted for a median of 2 days. These results suggest that children with NoV detected are probably brought to the hospital for the treatment of numerous vomiting episodes rather than severe dehydration or diarrhoea. In NoV challenge studies, vomiting was more prevalent than diarrhoea and nearly half of the participants who experienced vomiting did not report diarrhoea symptoms [30]. Based on the surveillance case definition, $\mathrm{NoV}$ cases presenting as only vomiting or treated in the outpatients department will have been missed. Furthermore, a large proportion of children with NoV may not have required medical treatment resulting in an under estimation of the true prevalence of NoV diarrhoea in children $<5$ years in SA.

NoV GI strains have been detected at similar levels to GIIs in river water in SA [28] but were not seen at comparable levels in hospitalized patients $(12 \%$ vs. 3\%). These results suggest that either NoV GI strains survive longer in the environment or patients shed NoV GI strains at higher titres [31] or NoV GI strains cause less severe or asymptomatic infections. Furthermore, these cases may be treated at home or at a clinic level, which means that they would not be captured by the study surveillance system.

The exception to the perceived mild nature of GI infections may be in HIV-infected children. The current study indicated increased detection of single GI infections in HIV-infected children compared with HIV-uninfected children ( $9 \%$ vs. $1 \%)$ in children admitted for the treatment of diarrhoea. In addition, the median NoV GI $C_{\mathrm{t}}$ values in HIV-infected children were also significantly lower compared with HIVuninfected children $(P=0 \cdot 05)$. These results combined could be used as an indicator of NoV GI disease severity in HIV-infected children. Similar differences in disease severity in RV and NoV GII infections in HIV-infected and HIV-uninfected patients have not been reported. However, this may be due to our inability to distinguish subtle differences in clinical severity between the two populations rather than the absence i.e. diarrhoea infections associated with RV and NoV GII strains are severe regardless of immune status.

A study by Groome and Madhi [32] estimated that $26 \%$ of children admitted with acute gastroenteritis to
CHBAH, Johannesburg between March 1998 and October 2000 were HIV infected, based on the prevalence rate in women attending antenatal clinics in the area. While the study did not detect more frequent RV infections in HIV-infected children, the absolute burden of disease in these children was twofold higher than in HIV-uninfected children [32]. In addition, HIV-infected children were more likely to be hospitalized for a longer period and had a higher case fatality rate [32]. A similar trend was noted in the current study where NoV strains were detected at similar levels in HIV-infected and HIV-uninfected children. However, NoVs were associated with a higher case fatality rate in HIV-infected children compared with HIV-uninfected children.

Population denominators were unavailable for the surveillance sites and; therefore, the incidence and increased risk of NoV associated with HIV infections could not be calculated. However, the mean HIV prevalence among children $0-4$ years with NoV detected was 9.7\% (43/443) between 2009 and 2013 while the HIV prevalence among children $0-4$ years in the general population was $3 \cdot 3 \%$ in 2008 [33], a threefold increase. These results suggest that HIV infection may be associated with hospitalization of NoV cases in children and further study may be warranted.

Analysis of the environmental features associated with NoV detection found that NoVs were less common in diarrhoeal patients living in informal housing (aOR $0.7 ; P=0.021$ ) compared with brick housing or using external water sources (aOR $0.7 ; P=0.045$ ) compared with indoor water. The role of continuous environmental exposure to NoV strains in these settings is unclear. Future study including control groups from the same community without diarrhoea would be required to interrogate these findings further.

The study has several limitations that should be considered when evaluating the findings. Missing data were dealt with by pairwise deletion and information selection bias may be present affecting the estimates and associations observed. The comparison groups used for the analysis were not strict control groups with the absence of diarrhoea and may have resulted in an underestimation of the clinical and environmental features associated with NoV infections. While patients who refused to participate in the study were noted, no additional information was gathered from these patients and, therefore, there may be non-participation biases unaccounted for in the analysis. The study enrolled children who were 
admitted to hospital overnight and was limited to moderate-to-severe diarrhoea. Therefore, any findings are restricted to this category of diarrhoeal infections and should be extrapolated to less severe outcomes with care. Not all the participants included in the study had specimens screened for all enteric pathogens. Limited clinical specimen volumes resulted in reduced screening for parasites. This may have resulted in an underestimation of mixed infections and affected the analysis of single pathogen infections.

Asymptomatic infections and prolonged shedding of enteric pathogens complicates epidemiological evaluations when trying to establish whether a pathogen is associated with an illness. Asymptomatic NoV infections have been described in paediatric patients at frequencies between $11 \cdot 6 \%$ and $49 \cdot 2 \%$ in a recent review [6]. An Australian study recorded NoV GII shedding in seven young children for 2-100 days [34]. In addition, NoV shedding in immunocompromised patients has been recorded for up to 898 days [35]. The current study did not have any data on asymptomatic NoV infections or duration of NoV shedding and the frequency of mixed pathogen NoV cases ranged from $77 \%$ to $66 \%$.

However, efforts have been made to translate the faecal NoV viral load measurements or $C_{\mathrm{t}}$ values as a proxy measure into disease-attribution cut-offs [36]. An English study calculated an optimal $C_{\mathrm{t}}$ cut-off for children $<5$ of 30 [36]. As no South African $C_{\mathrm{t}}$-disease-attribution analysis of the $\mathrm{NoV}$ real-time detection assay has been performed, the current study included all specimens positive for NoV irrespective of $C_{\mathrm{t}}$ value. An interesting finding was the statistically significant difference in the median $C_{\mathrm{t}}$ values between sole pathogen and mixed pathogen cases $(27.2$ vs. $32.0 ; P=0.006)$. Additional research establishing the $C_{\mathrm{t}}$-disease-attribution cut-off and investigating NoV shedding in SA should be considered.

The study determined the detection rate, clinical characteristics and environmental features associated with NoV detection in hospitalized children $<5$ years in SA. Furthermore, the study identified NoV GI strains as a potentially serious pathogen in vulnerable HIV-infected patients and demonstrated an association between NoV detection and mortality in this group. Future monitoring of NoV detection rates and variants circulating in the South African population may aid in enumerating diarrhoea burden due to the introduction of new NoV strains.

\section{SUPPLEMENTARY MATERIAL}

The supplementary material for this article can be found at https://doi.org/10.1017/S0950268817000668.

\section{ACKNOWLEDGEMENTS}

The authors acknowledge participants and staff of the sentinel diarrhoea surveillance programme. Meera Chagan (deceased 29 August 2014) for supervision of the Kwa-Zulu Natal site, The South African Field Epidemiology Training Programme.

The sentinel diarrhoea surveillance programme was funded by GlaxoSmithKline (E-Track 200238). The funders were not involved in study design, writing or publication of the paper.

\section{DECLARATION OF INTERESTS}

N.A.P. received honoraria from GlaxoSmithKline, Merck and Aspen Pharma. M.J.G. received honoraria from GlaxoSmithKline and Sanofi Pasteur. C.C. received honoraria from Sanofi Pasteur and Pfizer.

\section{ETHICAL STANDARDS}

The authors assert that all procedures contributing to this work comply with the ethical standards of the relevant national and institutional committees on human experimentation and with the Helsinki Declaration of 1975, as revised in 2008. Ethical approval for the study was obtained from the Human Research Ethics Committee (Medical), University of Witwatersrand (M091018), the Biomedical Research Ethics Committee, University of Kwa-Zulu Natal (BF074/09) and the Faculty of Health Sciences Research Ethics Committee, University of Pretoria (278/2015).

\section{REFERENCES}

1. Ahmed SM, et al. Global prevalence of norovirus in cases of gastroenteritis: a systematic review and meta-analysis. Lancet Infectious Diseases 2014; 14: 725-730.

2. Patel MM, et al. Noroviruses: a comprehensive review. Journal of Clinical Virology 2009; 44: 1-8.

3. Pires SM, et al. Aetiology-specific estimates of the global and regional incidence and mortality of diarrhoeal diseases commonly transmitted through food. PLoS ONE 2015; 10(12): e0142927.

4. Patel MM, et al. Systematic literature review of role of noroviruses in sporadic gastroenteritis. Emerging Infectious Diseases 2008; 14: 1224-1231. 
5. Koopmans M. Progress in understanding norovirus epidemiology. Current Opinion in Infectious Diseases 2008; 21: $544-552$.

6. Robilotti E, Deresinski S, Pinsky BA. Norovirus. Clinical Microbiological Reviews 2015; 28: 134-164.

7. Hall AJ, et al. Norovirus disease in the United States. Emerging Infectious Diseases 2013; 19: 1198-1205.

8. Lopman B, et al. Global Burden of Norovirus and Prospects for Vaccine Development. Atlanta, GA, USA: Centre for Disease Control and Prevention and Centre for Disease Control and Prevention Foundation, August 2015 (https://www.cdc.gov/norovirus/downloads/global-burden-report.pdf). Accessed 26 January 2017.

9. Mans J, et al. Norovirus epidemiology in Africa: a review. PLoS ONE 2016; 11(4): e0146280.

10. Taylor MB, et al. Two successive outbreaks of SRSV-associated gastroenteritis in South Africa. Journal of Medical Virology 1993; 41: 18-23.

11. Taylor MB, et al. An epidemiological investigation of Norwalk virus infection in South Africa. Epidemiology and Infection 1996; 116: 203-206.

12. Smit TK, et al. Seroepidemiological study of genogroup I and II calicivirus infections in South and southern Africa. Journal of Medical Virology 1999; 59: 227-231.

13. Wolfaardt M, et al. Incidence of human calicivirus and rotavirus infection in patients with gastroenteritis in South Africa. Journal of Medical Virology 1997; 51: 290-296.

14. Rouhani S, et al. Norovirus infection and acquired immunity in 8 countries: results from the MAL-ED study. Clinical Infectious Diseases 2016; 62: 1210-1217.

15. Wingfield T, et al. Chronic norovirus infection in an HIV-positive patient with persistent diarrhoea: a novel cause. Journal of Clinical Virology 2010; 49: 219-222.

16. Rodríguez-Guillén $\mathbf{L}$, et al. Calicivirus infection in human immunodeficiency virus seropositive children and adults. Journal of Clinical Virology 2005; 33: 104-109.

17. Cegielski JP, et al. Enteric viruses associated with HIV infection in Tanzanian children with chronic diarrhea. Pediatric AIDS and HIV Infection 1994; 5: 296-299.

18. Van Stelten A, et al. Optimization of a real-time RT-PCR assay reveals an increase of genogroup I norovirus in the clinical setting. Journal of Virological Methods 2011; 175: 80-84.

19. Loisy F, et al. Real-time RT-PCR for norovirus screening in shellfish. Journal of Virological Methods 2005; 123: $1-7$.

20. Kageyama T, et al. Broadly reactive and highly sensitive assay for Norwalk-like viruses based on real-time quantitative reverse transcription-PCR. Journal of Clinical Microbiology 2003; 41: 1548-1557.

21. Groome MJ, et al. Effectiveness of monovalent human rotavirus vaccine against admission to hospital for acute rotavirus diarrhoea in South African children: a case-control study. Lancet Infectious Diseases 2014;
14: 1096-1104. Erratum in: Lancet Infectious Diseases 2014; 14: 1040 .

22. Samra NA, et al. Genetic characterization of Cryptosporidium spp. in diarrhoeic children from four Provinces in South Africa. Zoonoses and Public Health 2013; 60: 154-159.

23. Mans J, et al. Emerging norovirus GII.4 2008 variant detected in hospitalised paediatric patients in South Africa. Journal of Clinical Virology 2010; 49: 258-264.

24. Mounts AW, et al. Cold weather seasonality of gastroenteritis associated with Norwalk-like viruses. Journal of Infectious Diseases 2000; 181: S284-S287.

25. Raboni SM, et al. Acute gastroenteritis and enteric viruses in hospitalized children in southern Brazil: aetiology, seasonality and clinical outcomes. Memórias do Instituto Oswaldo Cruz 2014; 109: 428-435.

26. Lopman BA, et al. A summertime peak of "winter vomiting disease": surveillance of noroviruses in England and Wales, 1995 to 2002. BMC Public Health 2003; 24(3):13.

27. Boga JA, et al. Etiology of sporadic cases of pediatric acute gastroenteritis in Asturias, Spain, and genotyping and characterization of norovirus strains involved. Journal of Clinical Microbiology 2004; 42: 2668-2674.

28. Mans J, et al. Diverse norovirus genotypes identified in sewage-polluted river water in South Africa. Epidemiology and Infection 2013; 141: 303-313.

29. Mans J, et al. Norovirus diversity in children with gastroenteritis in South Africa from 2009 to 2013: GII.4 variants and recombinant strains predominate. Epidemiology and Infection 2016; 144: 907-916.

30. Kirby AE, et al. Vomiting as a symptom and transmission risk in norovirus illness: evidence from human challenge studies. PLoS ONE 2016; 11(4): e0143759.

31. Kirby AE, et al. Disease course and viral shedding in experimental Norwalk virus and Snow Mountain virus infection. Journal of Medical Virology 2014; 86: 2055-2064.

32. Groome MJ, Madhi SA. Five-year cohort study on the burden of hospitalization for acute diarrhoeal disease in African HIV-infected and HIV-uninfected children: potential benefits of rotavirus vaccine. Vaccine 2012; 27(30): A173-A178.

33. Shisana O, et al. South African National HIV Prevalence, Incidence, Behaviour and Communication Survey, 2008: The Health of Our Children. Cape Town: HSRC Press, 2010.

34. Kirkwood CD, et al. Calicivirus shedding in children after recovery from diarrhoeal disease. Journal of Clinical Virology 2008; 43: 346-348.

35. Schorn R, et al. Chronic norovirus infection after kidney transplantation: molecular evidence for immune-driven viral evolution. Clinical Infectious Diseases 2010; 51: 307-314.

36. Phillips G, et al. Diagnosing norovirus-associated infectious intestinal disease using viral load. BMC Infectious Diseases 2009; 9: 63. 